\title{
Blowout of Nonpremixed Flames: Maximum Coaxial Air Velocities Achievable, with and without Swirl
}

\author{
DOUGLAS FEIKEMA, RUEY-HUNG CHEN, and JAMES F. DRISCOLL* \\ Department of Aerospace Engineering, The University of Michigan, Ann Arbor, MI 48109
}

\begin{abstract}
The present study demonstrates how to optimize parameters in order to maximize the amount of coaxial air that can be provided to a nonpremixed jet flame without causing the flame to blow out. Maximizing the coaxial air velocity is important in the effort to reduce the flame length and the oxides of nitrogen emitted from gas turbines and industrial burners, a majority of which use coaxial air. Previous measurements by the latter two authors have shown that a sixfold reduction in the $\mathrm{NO}_{x}$ emission index of a jet flame is possible if sufficient coaxial air can be provided without blowing the flame out. The coaxial air shortens the flame and forces the reaction zone to overlap regions of higher gas velocity, which reduces the residence time for $\mathrm{NO}_{x}$ formation. The present work concentrates on demonstrating ways to prevent flame blowout when the following two constraints are imposed: (1) the coaxial air velocities must be sufficient to shorten the flame to a specified length (in order to reduce $\mathrm{NO}_{x}$ emissions) and (2) the coaxial air flow rate must be sufficient to complete combustion without the need for ambient air, which is a common practical constraint. The zero swirl case is considered first, and the effects of adding swirl are measured and directly compared. The following were systematically varied: fuel velocity, air velocity, fuel tube diameter, air tube diameter, fuel type, and swirl number.

Measurements demonstrate that coaxial air alone (with zero swirl) can cause up to a twofold reduction in flame length. However, the flame is stable only if the velocity-to-diameter ratio of the fuel jet does not exceed a critical value. It is found that the addition of swirl improves the maximum-air blowout limits by as much as a factor of 6 . The results identify a strain parameter, based on the ratio of air velocity to air tube diameter $\left(U_{A} / d_{A}\right)$, which collapses the blowout curves for ten different conditions (burner size, swirl number) approximately to a single curve. A physical mechanism that explains the swirl flame data is presented. Swirl is believed to be beneficial because it reduces the local velocities, and thus the local strain rates, near the forward stagnation point of the recirculation vortex, where the flame is stabilized.
\end{abstract}

\section{NOMENCLATURE}

AF stoichiometric air-to-fuel mass ratio

$c_{1}, c_{2}$ constants that depend on fuel type

$d_{F}, d_{A}$ diameter of fuel tube and air tube, respectively

$L_{f} \quad$ flame length

$S \quad$ swirl number

$S_{L} \quad$ maximum laminar burning velocity for a given fuel type

$U_{\mathrm{A}}, U_{\mathrm{F}}$ initial axial velocity of air and fuel, respectively

\section{Greek Symbols}

$\alpha \quad$ thermal diffusivity

$\beta, \eta, \xi$ parameters defined in Eq. 2

\footnotetext{
* To whom all correspondence should be sent.
}

$\phi_{0} \quad$ overall equivalence ratio based on fuel and air flowrates

$\rho_{A}, \rho_{F}$ density of air and fuel, respectively

\section{INTRODUCTION}

Previously, the present authors have reported the maximum fuel velocity blowout limits of a jet flame surrounded by coaxial air [1]. In particular, the case of zero swirl velocity has been compared to the case for which swirl is imparted to the coaxial air. In contrast, the present study concentrates on a different flame blowout limit, namely the maximum air velocity limit, rather than the maximum fuel velocity limit. At the maximum air velocity limit, the flame has overall fuel-lean conditions. There are several practical reasons why it is desirable to achieve overall fuel-lean conditions and thus maximize the air velocity, while avoiding flame blowout. Chen and Driscoll 
[2] have shown that it is possible to achieve a significant reduction of the $\mathrm{NO}_{x}$ emission index for a jet flame if sufficient coaxial air is provided to shorten the flame. In their case, a sixfold reduction of $\mathrm{NO}_{x}$ emission index was achieved. The reason that coaxial air shortens the flame is that the addition of air into the initial jet stream reduces the amount of air that must be entrained downstream in order to dilute the fuel to stoichiometric proportions [3]. By using coaxial air to shorten the flame, the entire flame zone moves upstream and therefore experiences fluid velocities which are relatively larger, which causes a reduction in the residence time for thermal $\mathrm{NO}_{x}$ to be produced. However, to achieve these $\mathrm{NO}_{x}$ reductions, a relatively large velocity of the coaxial air is required, so that it is necessary to optimize the diameters and the velocities of the fuel and the coaxial air to prevent flame blowout.

It is noted that virtually all gas turbine engines and all low $\mathrm{NO}_{x}$ nonpremixed industrial flames use coaxial air, and most add swirl to the coaxial air. With gas turbine engines (some of which are stationary and use gaseous fuels, as used in the present study), the excessive-air velocity blowout limit is a serious problem. When the gas turbine fuel flow rate is reduced, the air velocity tends to decrease less rapidly than the fuel velocity because of the inertia of the compressor, and the excessive coaxial air velocity causes the flame to blow out.

The purpose of the present article is to describe two methods to maximize the coaxial air velocity, and therefore shorten the flame length without blowing the flame out. With the first method, coaxial air with zero swirl is used. The reduction in flame length is measured and some measured blowout limits are compared with the predictions of the analysis by Broadwell et al. [3] and Dahm and Mayman [4]. The proper parameters that will minimize the flame length are discussed. The second step is to add swirl to the coaxial air, which provides significant additional flame stabilization. Swirl creates a recirculation vortex that interacts with the fuel jet [5-7], causing a flame-vortex interaction. In a previous article, the authors reported the effects of swirl on the maximum fuel velocity limits [1]. Such flames were lifted prior to blowout. The present work considers a different blowout limit, one that is associated with an excessive coaxial air velocity, rather than an excessive fuel velocity. At the maximum air velocity blowout limits studied herein, the flame blows out suddenly without lifting off.

The physical mechanisms that control the liftoff and blowout of simple jet flames have been described by three different theories [3, 4, 8-10]. In all three, it is agreed that the base region of a flame is extinguished if it is forced to overlap a region of excessively large local (and instantaneous) flow velocity. The local criteria to be applied is in dispute. Kalghatgi [8] argues that in a lifted flame local premixing occurs. His flame stability criterion is that at some point the local turbulent burning velocity must exceed the local flow velocity. Peters [10] instead describes the initial liftoff process by considering a nonpremixed flame that exists at the instantaneous stoichiometric contour. His stability criterion is that the scalar dissipation rate at the stoichiometric contour cannot exceed a critical value. Broadwell, et al. [3] postulate that the flame base cannot be stabilized if the quantity $U / \delta$ sufficiently exceeds the characteristic chemical reaction rate $S_{L}^{2} / \alpha$, where $S_{L}$ is the maximum laminar burning velocity, $\alpha$ is the thermal diffusivity, $U$ is local centerline velocity, and $\delta$ is the local jet width. $U / \delta$ can be interpreted as a mixing rate or as a local strain rate. By relating $U$ and $\delta$ to jet exit conditions, they find that the fuel velocity at blowout $\left(U_{F}\right)$ for a simple jet flame with no coaxial air is

$U_{F}=d_{F}\left(S_{L}^{2} / \alpha\right) c_{1}$,

where $d_{F}$ is the fuel jet diameter and $c_{1}$ is a constant that depends on fuel type. Both of the analyses of Kalghatgi [8] and Broadwell et al. [3] predict a result that is of the form given by Eq. 1, which has been verified by experiment. Attempts to determine which local flame concept is most realistic have been limited by the need to simultaneously measure the instantaneous flame base position as well as the instantaneous degree of premixing ahead of the flame, which requires Kolmogorov scale resolution. Sufficient data to assess the degree of local premixing are not yet available.

The effects of adding coaxial air have been considered in the analysis of Dahm and Mayman 
[4]. The Dahm-Mayman (D-M) analysis views the jet from the far field and treats the fuel jet and coaxial air as a hypothetical single jet source having the same total momentum as the fuel jet-coaxial air combination. The D-M analysis correctly predicts that coaxial air shortens and destabilizes the flame. By adding coaxial air, less ambient air is needed to dilute the fuel to stoichiometric proportions, so the flame is shortened. The following is a physical interpretation that explains why coaxial air destabilizes the flame. Coaxial air shortens the flame, which forces the entire reaction zone, from the lifted flame base to the flame tip, to move upstream and overlap regions of relatively higher velocity and strain. When the coaxial air has moved the lifted flame base so far upstream that $U / \delta$ exceeds $S_{L}^{2} / \alpha$ as discussed above, the flame blows out. In addition, adding coaxial air increases the local gas velocity at any axial location, which is a destabilizing effect.

The D-M analysis is based on the finding that blowout occurs when the local centerline value of $U / \delta$ equals $0.21 S_{L}{ }^{2} / \alpha$ [3]. The local centerline velocity $U$ is related to the initial fue.' and air velocities by equating the total momentum flux at any location, which scales as $\rho U^{2} \delta^{2}(\pi / 4)$ to the initial momentum flux $\rho_{F} U_{F}^{2} d_{F}{ }^{2}(\pi / 4)+$ $\rho_{A} U_{A}{ }^{2}\left(d_{A}{ }^{2}-d_{F}{ }^{2}\right)(\pi / 4)$. Combining these concepts, their analysis predicts that the coaxial air velocity $\left(U_{A}\right)$ that causes blowout for a given fuel velocity $\left(U_{F}\right)$ is

$$
U_{A}^{2}=\xi \beta^{4 / 3} \eta d_{A}{ }^{2 / 3} U_{F}^{4 / 3}-\beta \eta U_{F}^{2} .
$$

The parameter $\xi$ is $\left[\left(S_{L}^{2} / \alpha\right)(1+\mathrm{AF})^{2} /\right.$ $4.8]^{2 / 3}$, where AF is the stoichiometric air-fuel mass ratio, which is $\mathbf{1 7 . 2}$ for methane. The parameter $\beta$ is $\left(\rho_{F} / \rho_{A}\right)\left(d_{F} / d_{A}\right)^{2}, \eta$ is $1 /[1-$ $\left(d_{F} / d_{A}\right)^{2}$, and $d_{A}$ is the coaxial air tube diameter. The initial velocity profiles are assumed to be uniform in deriving Eq. 2; however, only a 7\% difference in the value of $U_{A}$ is predicted when fully developed turbulent pipe flow profiles are used instead. The thermal diffusivity $\alpha$ in the analysis corresponds to stoichiometric elevated temperature conditions and is typically $4.5 \mathrm{~cm}^{2} / \mathrm{s}$. When swirl is added to the coaxial air, none of the above analyses apply directly. Previously there has been insufficient flame blowout data available to guide the analysis. For swirl-stabilized flames at the maximum air velocity limit, measurements have been reported in Refs. 11 and 12 for a relatively narrow range of conditions. A systematic series of measurements obtained for flames having swirl and zero swirl are presented below and are compared.

\section{EXPERIMENTAL ARRANGEMENT}

Figure 1a shows a schematic of the jet flame arrangement when coaxial air with zero swirl was used. The fuels used were methane, hydrogen, or combinations of the two. The fuel passes through an inner tube which has inner diameter $d_{F}$; three geometrically similar burners were used that have $d_{F}$ equal to $0.22,0.34$, and $0.48 \mathrm{~cm}$. Coaxial air

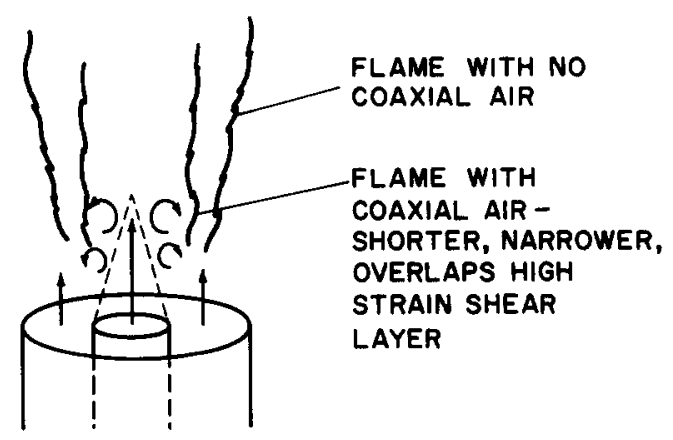

(a) ZERO SWIRL

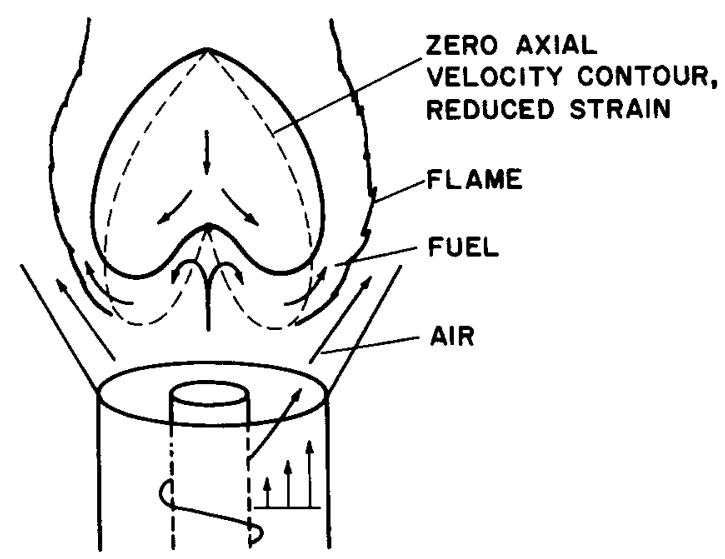

(b) WITH SWIRL

Fig. 1. Effect of coaxial air on flame base with (a) zero swirl and (b) swirl. Solid line in part (b) is recirculation vortex dividing streamline, averaged over time. 
is injected axially through an outer tube whose inner diameter $\left(d_{A}\right)$ is $6.54 d_{F}$. When swirl was added to the coaxial air, the arrangement shown in Fig. 1b was used. Various degrees of swirl can be imparted to the air by mixing tangentially injected air with axial air upstream of the burner exit. A more detailed description of the apparatus is given in Ref. 5. A laser velocimeter was used to measure the throat swirl number $(S)$, which is proportional to the flux of angular momentum to the flux of axial momentum at the throat, as defined in Ref. 13. After calibration, the swirl number can be inferred directly from the measured flowrates of axial and tangential air [14]. To promote stronger flow recirculation and thus stabilize overall lean flames, the swirl burner sidewalls diverged from the axial direction by an angle of 30 degrees. Flowrates were metered to an accuracy of $5 \%$ using 15 calibrated choked orifices and four rotameters.

\section{RESULTS FOR ZERO SWIRL}

The maximum coaxial air velocity that can be achieved before flame blowout occurs, for a given fuel jet velocity, is plotted in Fig. 2. It is seen that the zero swirl curve in Fig. 2 defines a peninsula-shaped stable region. The blowout curve has a $y$-axis intercept is $61.7 \mathrm{~m} / \mathrm{s}$. This value represents the fuel velocity required to blow out a methane-air flame with no coaxial air having jet diameter $d_{F}$ of $0.34 \mathrm{~cm}$; it agrees with measurements reported by Kalghatgi [8] within an accuracy of $10 \%$. For a given air velocity, there exists a maximum and a minimum fuel velocity for which stable flames can be achieved. The maximum fuel velocity limit is explained by the same concept that is used to explain the blowout of jet flames with no coaxial air; increasing the fuel velocity increases the local strain rate $U / \delta$ directly until it sufficiently exceeds the reaction rate $S_{L}^{2} / \alpha$. The minimum fuel velocity limit occurs because reducing $U_{F}$ increases the relative amount of air in the initial jet flow. This shortens the flame and forces the lifted flame base to move upstream to a region of the higher local strain rates that exist near the nozzle. The dotted line in Fig. 2 represents the blowout curve predicted by Eq. 1, which results from the analysis of Ref. 4 . The predictions are in good agreement with the present measurements.

It is now described how to achieve a minimum

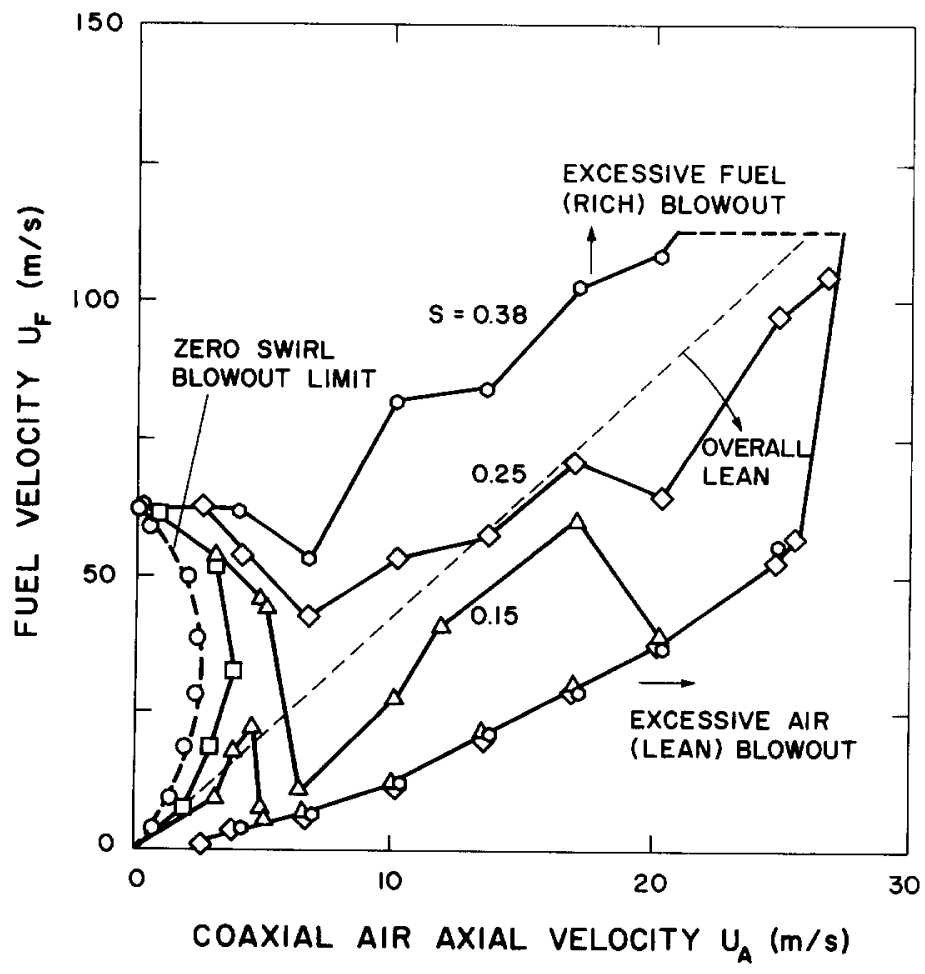

Fig. 2. Flame blowout limits for zero swirl compared with blowout limits with swirl. Methane fuel, $d_{A}=2.22 \mathrm{~cm}, d_{F}=0.034$ $\mathrm{cm}$. Swirl number: $O, S=0.0 ; \square, S=$ $0.10 ; \triangle, S=0.15 ; \diamond, S=0.25 ; \bigcirc, S=$ 0.38 . Dashed curved line near zero swirl data is theory of Ref. 4, as given by Eq. 2 . 
flame length by forcing a maximum amount of coaxial air into the flame. The proper choice of the operating parameters can be made by considering the relation for flame length $\left(L_{f}\right)$, which is derived in Ref. 4:

$$
\frac{L_{f} / d_{F}}{\left(L_{f} / d_{F}\right)_{0}}=\left[1+\frac{\rho_{A}}{\rho_{F}} \frac{U_{A}{ }^{2}}{U_{F}^{2}}\left(\frac{d_{A}{ }^{2}}{d_{F}{ }^{2}}-1\right)\right]^{-1 / 2} .
$$

$\left(L_{f} / d_{F}\right)_{0}$ is the normalized flame length for no coaxial air, which is $\mathbf{2 0 0}$ for methane-air turbulent jet flames [3]. To assess Eq. 3, the predicted and the measured flame lengths of hydrogen-air flames with coaxial air are plotted in Fig. 3; the agreement is satisfactory.

It is important to understand how to minimize the length of a flame in the effort to control nitric oxides. The minimum possible flame length $\left(L_{f}\right)_{\min }$ will be the flame length when the coaxial air causes blowout. Since Eq. 2 predicts the air velocity that causes blowout, it follows that the minimum flame length can be predicted by inserting the relation for $U_{A}{ }^{2}$ given by Eq. 2 into Eq.

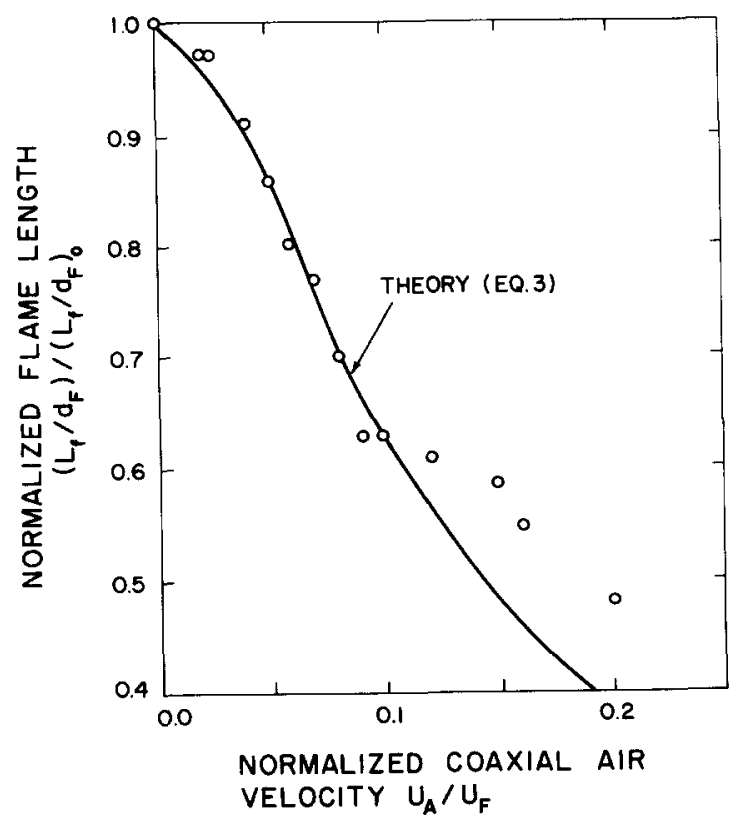

Fig. 3. Reduction in the length of a jet flame due to coaxial air (zero swirl) compared with prediction of Ref. 4. Fuel = Hydrogen, $\left(L_{f} / d_{F}\right)_{0}=168, d_{F}=0.26 \mathrm{~cm}, U_{F}=183 \mathrm{~m} / \mathrm{s}$, $\operatorname{Re}_{F}=5000, d_{A}=0.87 \mathrm{~cm}$.
3, which yields the following simple result:

$$
\left(L_{f} / d_{F}\right)_{\min } /\left(L_{f} / d_{F}\right)_{0}=\left(c_{2} U_{F} / d_{F}\right)^{1 / 3},
$$

where $c_{2}$ is a constant that depends only on fuel properties and equals $4.8\left(S_{L}{ }^{2} / \alpha\right)^{-1}(1+$ AF $)^{-2}\left(\rho_{F} / \rho_{A}\right)^{-1 / 2}$. Values of $c_{2}$ for hydrogen and methane fuels are 0.95 and $58.4 \mu \mathrm{s}$, respectively.

Experimental results shown in Fig. 4 confirm that Eq. 4 correctly predicts the minimum flame length that occurs at blowout. Several fuels were used. For each value of fuel velocity, the coaxial air velocity was increased until blowout occurred; just prior to blowout the flame length was measured visually. For example, Eq. 4 predicts that in order to halve the flame length of a methane-air flame by using coaxial air, the quantity $U_{F} / d_{F}$ must not exceed $(0.5)^{3} / c_{2}$ or $2140 \mathrm{~s}^{-1}$. Thus, a sufficiently small value of $U_{F} / d_{F}$ is required or else the flame will blow out before enough coaxial air can be forced into it such that the flame length is halved. This minimum value of $U_{F} / d_{F}$ equal to $2140 \mathrm{~s}^{-1}$ is very restrictive because in practical devices a minimum fuel velocity is re-

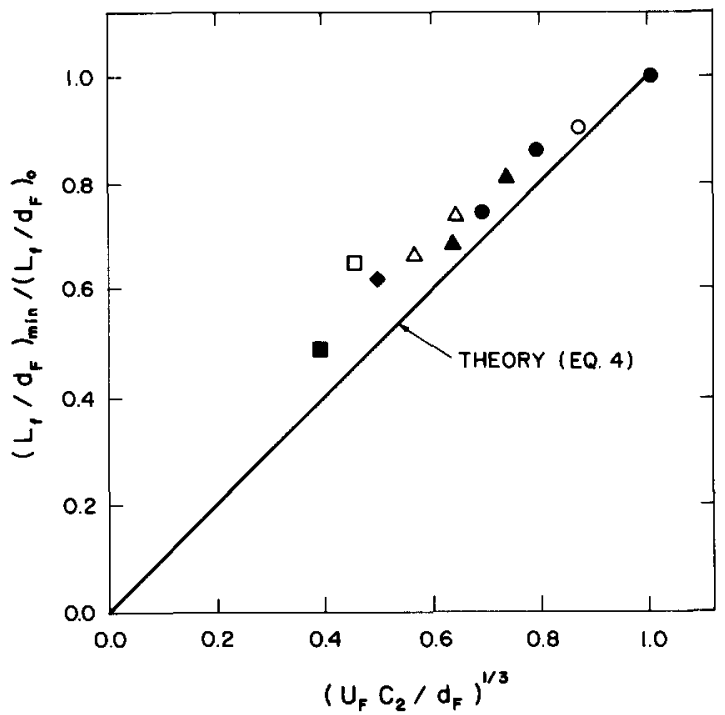

Fig. 4. Minimum flame length achievable by increasing coaxial air velocity until blowout occurs. Zero swirl. $\left(L_{f} / d_{F}\right)_{0}$ is jet flame length for no coaxial air and is equal to 200 for methane-air and 168 for hydrogen-air. $\mathrm{O}=\mathrm{CH}_{4}, d_{F}=0.26$ $\mathrm{cm}, \bullet=\mathrm{CH}_{4}, d_{F}=0.37 \mathrm{~cm}, \square=\mathrm{H}_{2}, d_{F}=0.26 \mathrm{~cm}$, $\mathbf{\square}=\mathrm{H}_{2}, d_{F}=0.37 \mathrm{~cm}, \Delta=0.5 \mathrm{CH}_{4}+0.5 \mathrm{H}_{2}, d_{F}=$ $0.37 \mathrm{~cm}, \bullet=0.75 \mathrm{H}_{2}+0.25 \mathrm{CH}_{4}, d_{F}=0.37 \mathrm{~cm}, \Delta=$ $0.33 \mathrm{H}_{2}+0.67 \mathrm{CH}_{4}, d_{F}=0.37 \mathrm{~cm}$. Constant $c_{2}$ given in Eq. 4. 
quired to meet the heating load requirements; to achieve the necessary fuel velocity, Eq. 4 would force the resulting fuel tube diameter $d_{F}$ and flame length to be excessively large. It is shown below that significantly larger values of $U_{F} / d_{F}$ are possible when swirl is used. This is one reason that coaxial air alone is not used in most practical devices that must be compact; instead, swirl is added to most gas turbine and industrial burner designs. It also is noted that because the value of $c_{2}$ for methane is 60 times larger than that for hydrogen, a methane flame is limited to a value of $U_{F} / d_{F}$ that is 60 times smaller than that of a hydrogen flame in order to achieve a similar reduction in flame length. To calculate the constant $c_{2}$ in Eq. 4, the laminar flame speeds of hydrogen-methane mixtures measured by $\mathrm{Yu}$ et al. [15] were used.

Another parameter that is of importance when stabilizing flames in coaxial air streams is the absolute maximum velocity above which no flame can be stabilized, regardless of the fuel velocity selected. This parameter is denoted $U_{A, \max }$. In Fig. 2 for example, the point that is farthest to the right on each curve defines $U_{A, \max }$. For zero swirl, the value of $U_{A, \max }$ is predicted by calculating the maximum value of $U_{A}$ in Eq. 2; differentiation yields

$$
\begin{aligned}
\left(U_{A, \max } / d_{A}\right) /\left(S_{L}{ }^{2} / \alpha\right) \\
=0.080(1+A F)^{2}\left(\rho_{F} / \rho_{A}\right)\left(d_{F} / d_{A}\right)^{2} \\
\quad \times\left(1-\left(\frac{d_{F}}{d_{A}}\right)^{2}\right)^{-1},
\end{aligned}
$$

where the parameters in Eq. 5 have been defined in Eq. 2. Equation 5 predicts that larger values of maximum air velocity can be achieved by selecting a larger value of the ratio of the fuel-to-air tube diameter ratio $d_{F} / d_{A}$. Measurements were made to assess this prediction; results are shown in Fig. 5. On the $y$ axis, the overall fuel-air equivalence ratio $\phi_{0}$ is plotted rather than fuel velocity; the two are proportional since $\phi_{0}=$ $\rho_{F} U_{F} d_{F}^{2}(\mathrm{AF}) /\left[\rho_{A} U_{A}\left(d_{A}^{2}-d_{F}^{2}\right)\right]$. The solid and dashed lines in Fig. 5 are the blowout curves predicted by Eq. 2, which are seen to agree with the present measurements. Fig. 5 also shows that by increasing the fuel-to-air diameter ratio

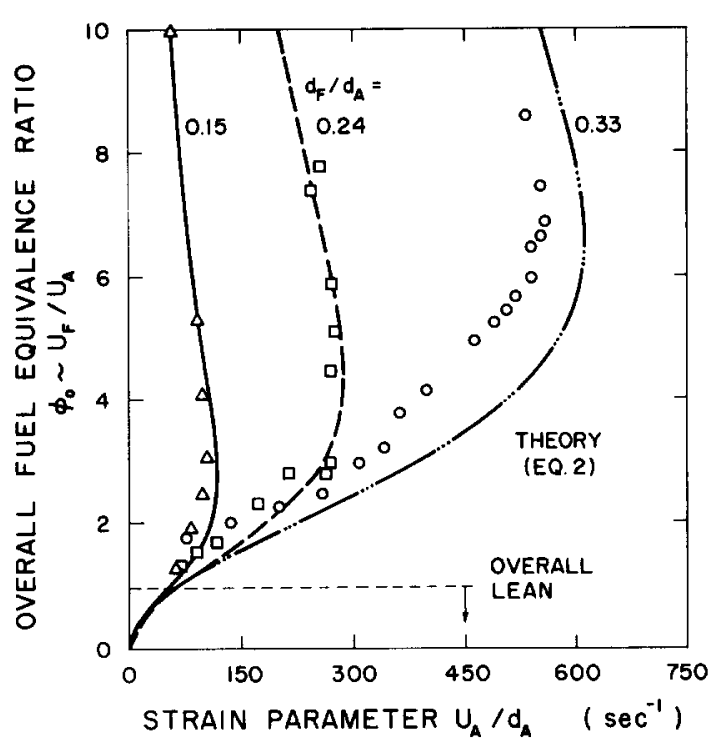

Fig. 5. Measured flame blowout limits of a methane-air jet flame with coaxial air and zero swirl, compared with predictions of Dahm-Mayman analysis.

$d_{F} / d_{A}$, the maximum air velocity increases significantly, in agreement with Eq. 5 .

The physical reason that selecting a larger value of the diameter ratio $d_{F} / d_{A}$ has a stabilizing effect is as follows. Coaxial air adds an additional axial velocity at any location on jet centerline. This additional velocity creates larger local values of $U / \delta$ (i.e., strain) which is destabilizing. Since the velocity on the centerline of a jet of diameter $d_{A}$ decays in the axial $(z)$ direction as $\left(d_{A} / z\right)$, it follows that reducing $d_{A}$ reduces the momentum of the air jet and therefore reduces the velocity contribution at the flame base due to the coaxial air. Thus, reducing $d_{A}$ increases $d_{F} / d_{A}$ and has a stabilizing influence, which is consistent with Fig. 5.

Equation 5 also predicts that $U_{A, \max }$ should vary linearly with burner size since the quantity $\left(d_{A}\right)$ appears in the denominator of the left-hand side of Eq. 5. Measurements of $U_{A, \max }$ were made to test the predicted effects of varying burner size. Results shown in Fig. 6 are in satisfactory agreement with the predicted scaling. The measurements were obtained by using coaxial fuel and air tubes having the same ratio of $d_{F} / d_{A}$ equal to 0.15 and $d_{A}$ equal to $1.44,2.22$, or 3.14 $\mathrm{cm}$. It is noted that Fig. 5 implies that it is very difficult to achieve an overall stoichiometric flame $\left(\phi_{0}=1\right)$ using coaxial air and no swirl. Using 


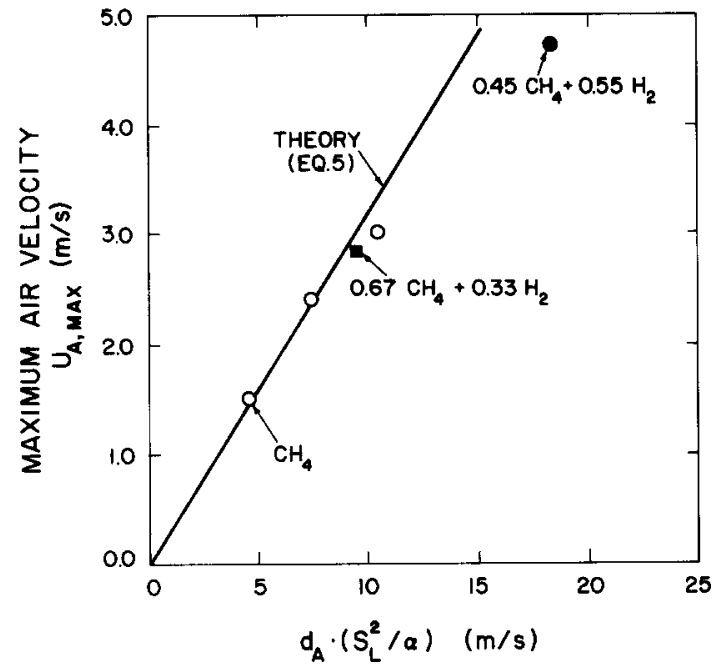

Fig. 6. Measured maximum coaxial air velocity at blowout for zero swirl, compared with predictions of Dahm-Mayman theory. Points represent maxima of curves of the type shown in Fig. 5.

methane fuel and the diameters chosen, Fig. 5 shows that the value of $U_{A} / d_{A}$ must be less than $80 \mathrm{~s}^{-1}$ to achieve $\phi_{0}=1.0$. The relative improvement in flame stability that can be achieved by adding swirl to the coaxial air is described in the next section.

\section{MAXIMUM COAXIAL AIR VELOCITY - WITH SWIRL}

Swirl was added to the coaxial air; also added was a diverging wall section that is shown in Fig. 1b. A diverging sidewall is commonly used in gas turbines and industrial burners. The diverging sidewalls strengthen the recirculation zone because they help to convert the axial vorticity vectors (i.e., the inlet swirl) into aximuthal vorticity (i.e., the recirculation vortex) for reasons discussed in Ref. 7 and in Batchelor's textbook [16]. The mean flowfield is shown schematically in Fig. 1b, which was deduced from data of Refs. 6 and 7. Details of the flowfield are discussed in the next section.

A photograph of a swirl-stabilized methane flame near the maximum coaxial air blowout limit appears as Fig. 7. The flame is described as ultralean because the overall fuel-air equivalence ratio is 0.14 . The flame is extremely short, having a normalized flame length $\left(L_{f} / d_{F}\right)$ of 20 ;

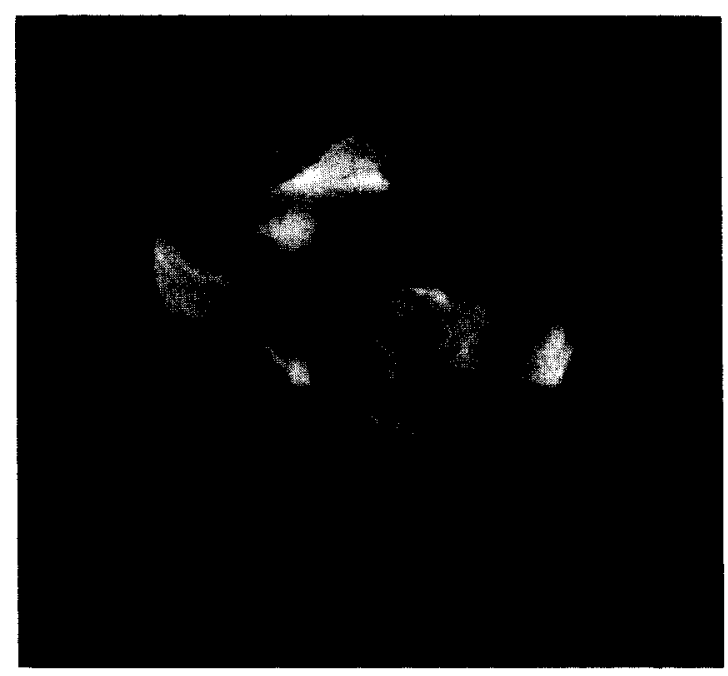

Fig. 7. Photograph of overall ultralean flame $\left(\phi_{0}=0.14\right)$ stabilized using swirl. Methane, $S=0.25, U_{A} / d_{A}=95 \mathrm{~s}^{-1}$. Note that flame is very short and fragmented.

without coaxial air or swirl the normalized flame length of a methane jet flame is 200 . Figure 7 shows that the flame stabilization point is about one $d_{F}$ downstream of the fuel tube, which is near the forward stagnation point of the recirculation vortex. Figure 7 shows that the flame surface is fragmented and discontinuous due to the fact that not much fuel is present, relative to the air flowrate, in the overall lean flame.

It is found that the swirl causes up to a sixfold improvement to the lean blowout limits, which is quantified by Fig. 2 . The maximum axial velocity of the air $\left(U_{A}\right)$ that can be achieved with no swirl is seen to be $2.4 \mathrm{~m} / \mathrm{s}$; for the same fuel flow rate and diameters $d_{A}$ and $d_{F}, U_{A}$ can be increased above $15 \mathrm{~m} / \mathrm{s}$ by operating at a swirl number of 0.38 . Figure 2 shows that a small degree of swirl ( $S=0.10$ ) produces a blowout curve that is similar to the zero swirl curve; dramatic improvements only occur for swirl number of 0.15 and above, at which point a recirculation zone is formed. Figure 2 also shows that if the flame must be operated in an environment having no ambient air (i.e., the coaxial air is the only air source) then swirl is required for flame stabilization, at least for the diameters $d_{A}$ and $d_{F}$ chosen. That is, to completely burn the fuel using coaxial air, one must operate to the right of the dashed straight line in Fig. 2, which is the region where the overall equivalence ratio is unity or 
less. Only the curves for swirl number of 0.15 and above lie in this overall lean region.

\section{PHYSICAL MECHANISM/CORRELATION OF SWIRL FLAME RESULTS}

The physical mechanism that is believed to be the reason that swirl causes a substantial improvement to the stability limits of overall lean flames can be explained with the aid of Fig. 1b. Swirl creates a zero axial velocity contour, shown in Fig. 1b, where the strain rates are relatively low. If the instantaneous flammable region overlaps the instantaneous zero axial velocity contour, the flame is believed to be stabilized. The term flammable region refers not only to the instantaneous surface where exact stoichiometry occurs, but to the strip of fluid that is molecularly mixed to concentrations that are within the flammability limit. This concept is consistent with the general flame liftoff/blowout concepts of Peters [10], Broadwell et al. [3], and others [11, 12]. Since the flame does not lift off, the theory of Kalghatgi, which requires that a long liftoff/premixing region exists, does not apply.
Consistent with the above idea, it is now shown that if parameters are varied so that the stoichiometric region is forced to move away from the zero axial velocity region, the experimental data indicate that the flame will be destabilized. It is first noted that near-field parameters are the important ones because the flames considered do not lift off into the far-field, but blow out suddenly when a critical air velocity is exceeded. The near-field flame extinction of the present flames therefore is believed to be due to strainout, just as the initial liftoff of a jet flame is controlled by strainout [10].

The two near-field parameters of importance are $U_{A} / d_{A}$ and $U_{F} / U_{A}$ since (1) the strain rate associated with the air flow depends on $U_{A} / d_{A}$ and the inlet air velocity profile, and (2) the location of the forward stagnation point depends on the ratio $U_{F} / U_{A}$. The location of the forward stagnation point occurs where the dynamic pressure of the fuel jet just balances the dynamic pressure of the reverse flow within the recirculation vortex. This balance yields an equation that is similar to that used to model a counterflow flame (see Eq. 9 in Ref. 17). The fuel jet dynamic

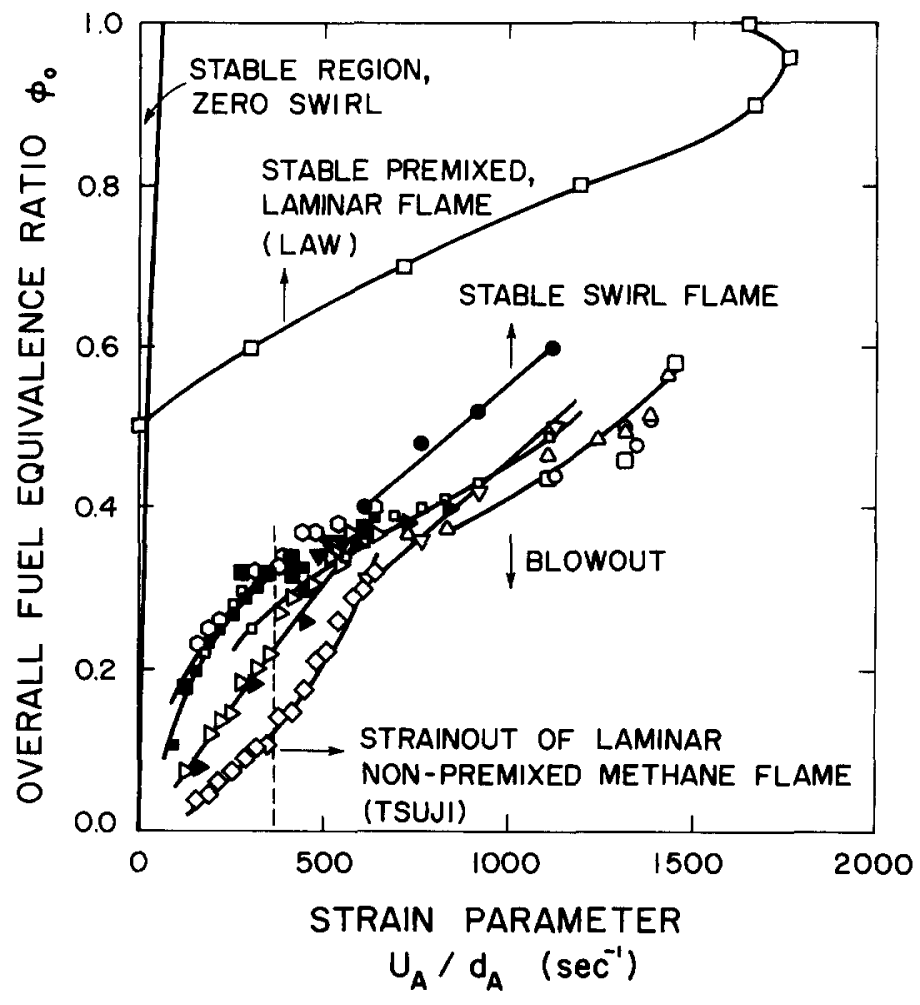

Fig. 8. Correlation of overall lean flame blowout limits with strain parameter $U_{A} / d_{A}$ using three burner sizes and several different swirl numbers. $U_{A}$ was varied for each burner and $d_{A}$ was varied by using three geometrically similar burners. Fuel-air diameter ratio is 0.15 and fuel is methane for all cases. For comparison, strain rates that extinguish other types of flames are shown. $d_{A}=1.44 \mathrm{~cm}$, $S=0.72: \triangle, 0.56: \square, 0.45: \circ ; d_{A}=2.22$ $\mathrm{cm}, S=1.1: \bullet 0.5: \nabla 0.375:-d_{A}=3.14$ $\mathrm{cm}, S=1.56 \diamond, 0.75: \triangleright, 0.25 \nabla, 0.175 \bigcirc$. 
pressure scales as $\rho_{F}\left[U_{F}\left(d_{F} / z\right)\left(\rho_{F} / \rho_{A}\right)^{1 / 2}\right]^{2}$, where the quantity in brackets is the centerline velocity of a jet. The reverse flow dynamic pressure scales as $\rho_{A} U_{A}^{2}$ for a fixed swirl number, since the recirculation zone reverse velocity scales with $U_{A}[5]$. Balancing these two dynamic pressures shows that the location of the stagnation point $\left(z / d_{F}\right)$ is proportional to $\left(U_{F} / U_{A}\right)$. In the present flames, $z / d_{F}$ was measured and was found to be proportional to $U_{F} / U_{A}[7]$.

Three trends can be predicted by using the above physical concepts. First, increasing $U_{A} / d_{A}$ should be destabilizing since the local strain rate would be increased. Secondly, increasing the normalized fuel velocity $U_{F} / U_{A}$ should be stabilizing. Increasing $U_{F} / U_{A}$ forces the forward stagnation point to move downstream; the fuel rich zone labeled in Fig. 1 moves downstream and away from the high-velocity air shear flow. The air velocities are maximum near the burner sidewalls due to the radially outward centrifugal forces. Therefore increasing $U_{F} / U_{A}$ should move the near-stoichiometric region downstream so that it overlaps a relatively lower strain rate region, which is stabilizing. A third trend that would be expected to occur is that if $U_{A} / d_{A}$ is a governing parameter, then by doubling the burner size, twice the air velocity will be required to blow out the flame, if the other parameter $\left(U_{F} / U_{A}\right)$ is held fixed.

The above three predicted trends are verified by the data of Fig. 8. Burner size was doubled and $U_{A}$ at blowout was found to double; by using the parameter $U_{A} / d_{A}$ ten different blowout curves approximately collapse to a single curve having positive slope. The positive slope in Fig. 8 is consistent with the trends predicted by the above physical concept. Increasing the global strain rate $\left(U_{A} / d_{A}\right)$ while proceeding along a horizontal line in Fig. 8 is observed to be destabilizing. Increasing the normalized fuel velocity $\left(U_{F} / U_{A}\right)$ while proceeding along a vertical line in Fig. 8 is observed to be stabilizing. Both observations are consistent with the above concepts.

Figure 8 shows that swirl allows overall ultralean flames to be stabilized. For zero swirl, the stable region on the left side of Fig. 8 is predicted by Eq. 2 to be a very restricted region in which $U_{A} / d_{A}$ is less than $80 \mathrm{~s}^{-1}$. With swirl, the flame is stable for $U_{A} / d_{A}$ up to $1500 \mathrm{~s}^{-1}$. Thus, for a given burner size, swirl allows much more air to be forced into the flame. It is concluded that to achieve ultralean flames have $\phi_{0}$ less than 0.2 , methane flames should be operated at relatively large swirl numbers of $0.75-1.56$ and $U_{A} / d_{A}$ should be less than $500 \mathrm{~s}^{-1}$.

The global strain rates associated with the present flame are compared with strain rates that extinguish other types of flames, as shown in Fig. 8. The comparisons are made to discuss orderof-magnitude trends only; it is realized that the local conditions and the local physics of different types of flame differ substantially. The extinction limit of a counterflow methane-air laminar nonpremixed flame was measured by Yamaoka et al. [18] to be $367 \mathrm{~s}^{-1}$. The global strain rates that are found to cause blowout in the present study are observed in Fig. 8 to be of the same orderof-magnitude as Yamaoka's value and to exceed his value in most cases. the extinction due to strain of a premixed, laminar, methane-air counterflow flame was studied by Law [19]; his results are plotted in Fig. 8. The strain rates that extinguish a premixed flame are of the same order-ofmagnitude as those measured for the present nonpremixed flame; it is noted that Law's results in Fig. 8 display a positive slope, as do the present results. However, direct comparison is not possible since the degree of partial premixing in the present case is not known.

Laser velocimetry and flow visualization methods were used to verify that a recirculation vortex does indeed occur in the present flames, and that low-velocity regions are created near the forward stagnation point where the flame is stabilized. A strong recirculation vortex was photographed within several of the present flames and appears in Ref. 6. The fuel flow is observed to impinge on the forward stagnation point and then to reverse its axial velocity, as depicted in Fig. 1b. Mean velocity vector diagrams and turbulence levels within the present flames as well as near the stagnation region of bluff body flames appear in Ref. 7 and are not repeated here. Figure 9 depicts the axial and tangential velocity profiles in the region just above the fuel tube for an isothermal case. At the lowest swirl number of 0.25 , the axial velocity profile is uniform, whereas at higher swirl numbers the flow is progressively thrown outward by centrifugal forces, creating a lowvelocity region near centerline that becomes a 


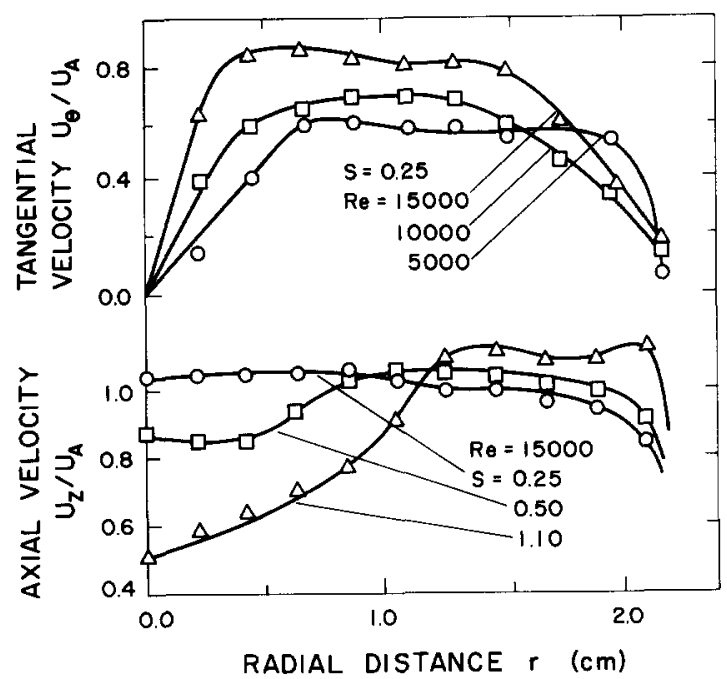

Fig. 9. Effect of swirl on the burner throat velocity profiles. Note that swirl causes a momentum deficit at radial inward positions, which is beneficial to flame stabilization.

stagnation point downstream. Although mean and root-mean-square (rms) velocity measurements near the flame base are useful, they cannot answer fundamental questions about the local stability criterion. Conditioned velocity measurements are needed at locations just ahead of the moving, unsteady flame base for a lifted, swirl-stabilized flame.

The effect of adding hydrogen to the methane fuel is shown in Fig. 10. The physical mechanism described previously predicts that hydrogen enriched flames, which have larger characteristic reaction rates $S_{L}^{2} / \alpha$ than pure methane flames, should withstand correspondingly larger strain rates $U_{A} / d_{A}$. Figure 10 verifies this trend; for example, if $\phi_{0}$ is set equal to 0.3 , the addition of $55 \%$ hydrogen to the methane fuel increases the strain rate $U_{A} / d_{A}$ that the flame can withstand from 300 to $2400 \mathrm{~s}^{-1}$. Another parameter that was varied was the fuel-to-air diameter ratio $d_{F} / d_{A}$. Results are shown in Fig. 11. Previously it was shown in Fig. 5 that increasing $d_{F} / d_{A}$ has a stabilizing influence on the zero swirl flames, as predicted by Eq. 5. With swirl, the data of Fig. 11 indicate that the same trend occurs. That is, selecting a larger value of $d_{F} / d_{A}$ also has a stabilizing effect on the swirl flames since the curves in Fig. 11 extend farther to the right as $d_{F} / d_{A}$ increases. The physical reason for this trend appears to be the same as that which was

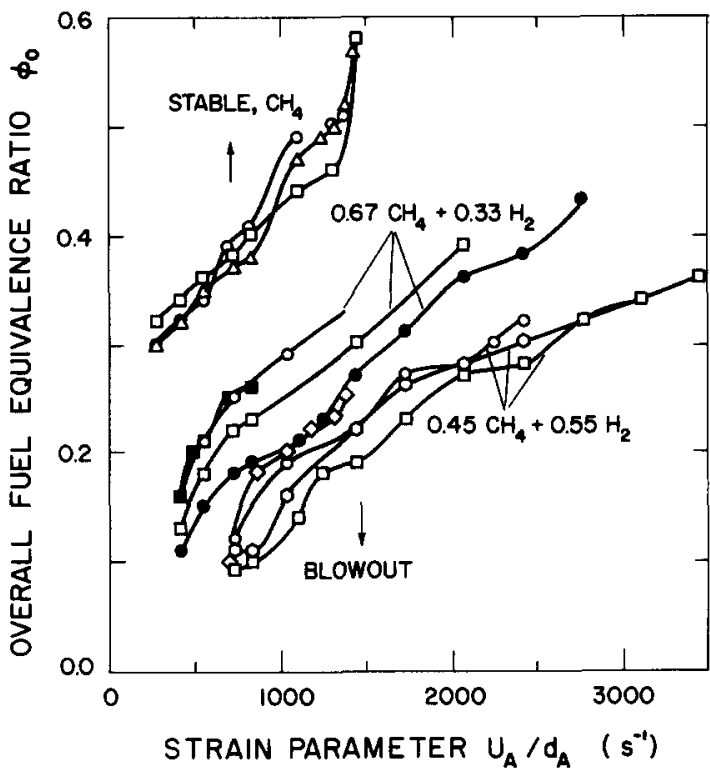

Fig. 10. Improvement to flame blowout limits due to hydrogen enrichment. $d_{A}=1.44 \mathrm{~cm} ; d_{F}=0.22 \mathrm{~cm} . S=0.72$ : $\triangle \bullet \square$; 0.5: $\square, 0.45: 0,0.375: \bigcirc, 0.325: \diamond$.

discussed for the zero swirl flames. Increasing $d_{F}$ is stabilizing, as shown by Eq. 1, whereas increasing $d_{A}$ is a destabilizing influence. Increasing the diameter of the coaxial jet $\left(d_{A}\right)$ increases the gas velocity that is induced by the air jet momentum at any $z$ location downstream on centerline; this induced velocity scales as $\left(d_{A} / z\right)$ - $U_{A}$ for the zero swirl case. Thus, selecting a larger value of $d_{A}$ will destabilize the flame. The general trends measured in the present study demonstrate that large changes in flame stability and flame length can be achieved by the use of coaxial air with or without swirl. Although no model yet exists that can successfully predict the swirl flame blowout limits, the measured trends reported herein could be used to assess future models.

\section{CONCLUSIONS}

1. For a jet flame surrounded by a coaxial air flow with no swirl, measurements were made of the minimum possible flame lengths and the corresponding maximum air velocities that occur just prior to flame blowout. An understanding of these limiting conditions is required in the effort to reduce nitric oxides and 


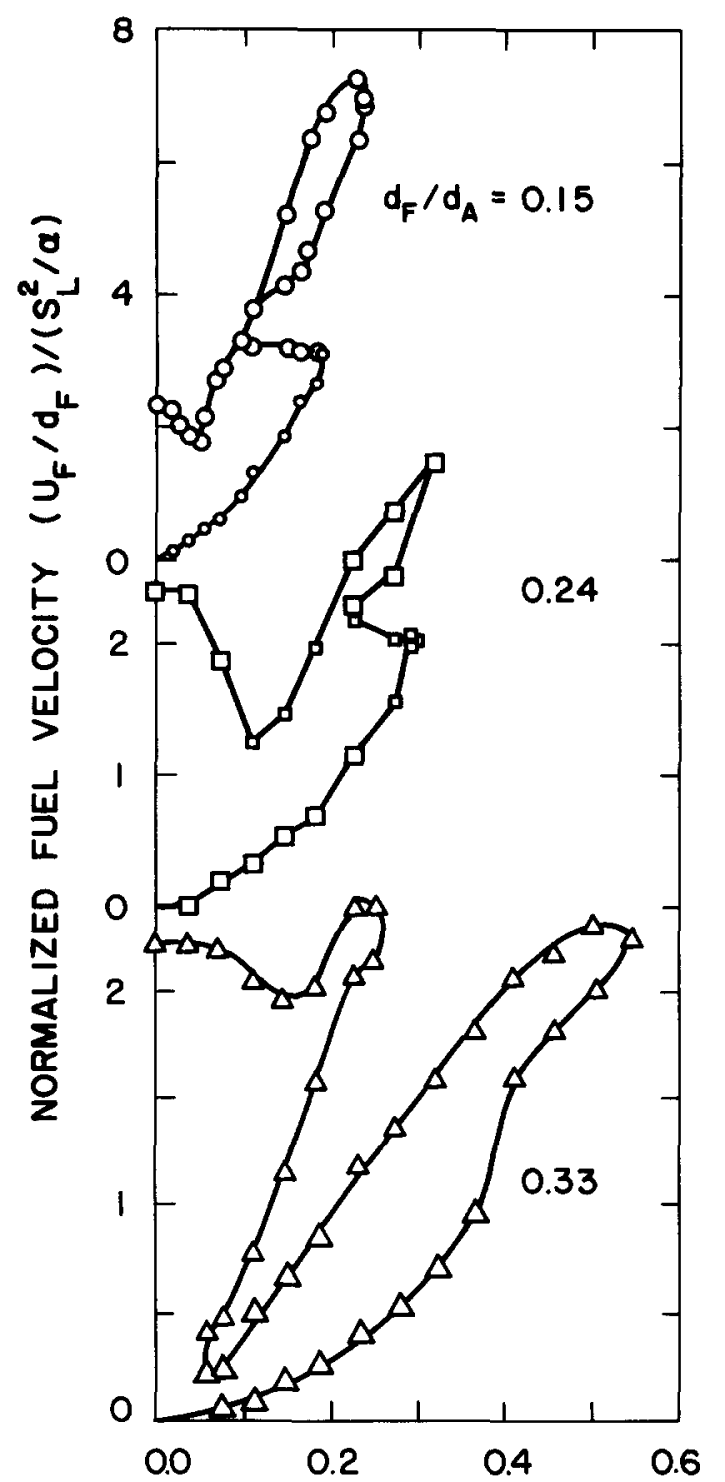

NORMALIZED AIR VELOCITY
$\left(U_{A} / d_{A}\right) /\left(s_{L}^{2} / a\right)$

Fig. 11. Effect of diameter ratio $\left(d_{F} / d_{A}\right)$ on swirl flame blowout limits. Methane fuel, $S=0.72$. Note that curves follow the same trend as the zero swirl curves Fig. 5 in that they extend farther to the right as $d_{F} / d_{A}$ increases.

to reduce combustor size. Trends were measured as burner size and fuel type were varied; the measurements show satisfactory agreement with the predictions of the DahmMayman [4] theory.

2. To shorten the jet flame by a desired amount by using coaxial air, both the measurements and the theory indicate that the ratio of fuel velocity to fuel tube diameter $\left(U_{F} / d_{F}\right)$ must be reduced below a critical value given by $\mathrm{Eq}$. 4 to prevent blowout.

3. To achieve an overall lean flame (i.e., if ambient air is not available and the only air for combustion is the coaxial air), and if no swirl is used, the flame blowout limits are shown to place severe restrictions on the velocities and diameters that can be chosen. With swirl, however, overall lean flames can be stabilized for a wide range of conditions.

4. It is believed that the reason that swirl enhances the stability of overall lean flames (which blow out but do not lift off) is that swirl creates a local region having a reduced velocity and a reduced local strain rate.

5. The maximum coaxial air blowout limits for swirl flames were obtained for three burner sizes and various swirl numbers. It was found that the governing parameter is the global strain rate $U_{A} / d_{A}$, that is, larger diameter burners will allow for operation at proportionally larger maximum air velocities. Using this parameter, ten different blowout curves approximately collapse to a single curve.

6. The effects of varying fuel type and fuel-to-air tube diameter ratio were measured for both zero swirl flames and for the swirl-stabilized flames. The trends agree with the physical concepts that describe flame stabilization. Both types of flames exhibited the same trends, namely improved stabilization occurred as the ratio $d_{F} / d_{A}$ is increased and as the maximum laminar burning velocity is increased.

This research is supported by the Gas Research Institute under contract 5087-260-1443; the contract monitors have been J. Kezerle and $T$. R. Roose. The authors acknowledge numerous useful discussions with Professor W. J. A. Dahm concerning his analysis.

\section{REFERENCES}

1. Feikema, D., Chen, R. -H., and Driscoll, J. F., Combust. Flame 80:183-195 (1990).

2. Chen, R. -H., and Driscoll, J. F., Twenty-Third Symposium (International) on Combustion, The Combustion Institute, Pittsburgh, 1990, p. 281.

3. Broadwell, J. E., Dahm, W. J. A., and Mungal, M. G., 
Twentieth Symposium (International) on Combustion, The Combustion Institute, Pittsburgh, 1985, p. 303.

4. Dahm, W. J. A., and Mayman, A. G., $A I A A J$. 28:1157-1162 (1990).

5. Tangirala, V., Chen, R. -H., and Driscoll, J. F., Combust. Sci. Technol. 51:75-95 (1987).

6. Chen, R. -H., and Driscoll, J. F., Twenty-Second Symposium (International) on Combustion, The Combustion Institute, Pittsburgh, 1989, p. 531.

7. Chen, R. -H., Driscoll, J. F., Kelly, J., and Namazian, M., Combust. Sci. Technol. 71:197-207 (1990).

8. Kalghatgi, G., Combust. Sci. Technol. 26:233-239 (1981).

9. VanQuickenborne, L., and van Tiggelen, A., Combust . Flame 10:59-68 (1966).

10. Peters, N., Twentieth Symposium (International) on Combustion, The Combustion Institute, Pittsburgh, 1984, p. 353.

11. Rawe, R., and Kremer, H., Eighteenth Symposium (International) on Combustion, The Combustion Institute, Pittsburgh, 1981, p. 667.
12. Leuckel, W., and Fricker, N., J. Inst. Fuel 49:152-158 (1976).

13. Ribiero, M. M., and Whitelaw, J. H., J. Fluid Mech. 96:769-795 (1980).

14. Claypole, T. C., and Syred, N., Eighteenth Symposium (International) on Combustion, The Combustion Institute, Pittsburgh, 1981, p. 81.

15. Yu, G., Law, C. K., and Wu, C. K., Combust. Flame 12:253-260 (1986).

16. Batchelor, G. K., Introduction to Fluid Mechanics, Cambridge University Press, Cambridge, 1967, Sec. 7.5 , p. 543 .

17. Darabiha, N., Candel, S. M., and Marble, F. E., Combust. Flame 64:203-217 (1986).

18. Yamaoka, I., Tsuji, H., and Harigaya, Y., Twenty-First Symposium (International) on Combustion, The Combustion Institute, Pittsburgh, 1986, p. 1837.

19. Law, C. K., Twenty-Second Symposium (International) on Combustion, The Combustion Institute, Pittsburgh, 1989, p. 1381.

Received 12 June 1990; revised 19 February 1991 